\title{
Suicide Attempt by Pacemaker System Abuse: A Case Report with Comments on the Psychological Adaptation of Pacemaker Patients
}

\author{
ARTHUR B. SIMON, PETER KLEINMAN, and NANCY JANZ \\ From the Department of Internal Medicine, Division of Cardiology, Department of Psychiatry, \\ Psychiatry Consultation Service and Nursing Services, University of Michigan Medical \\ Center, Ann Arbor, Michigan
}

\begin{abstract}
SIMON, A.B., KLEINMAN, P., AND JANZ, N: Suicide attempt by pacemaker system abuse: A case report with comments on the psychological adaptation of pacemaker patients. Thirty patients selected at random from a pacemaker clinic were interviewed to gain an understanding of how psychological symptoms in these patients related to their cardiac condition. In $20 \%$ veiled depression or even a wish to die was elicited. Joking, fantasies, and denial were common psychological mechanisms used to adapt to their illness and the pacemaker itself. A dramatic example of attempted suicide in which the patient tried to cut the subcutaneously implanted pacemaker leads is presented and illustrated. (PACE, Vol. 3, March-April, 1980)
\end{abstract}

suicide, pacemakers, psychosocial adjustment, depression

The psychological attitude of pacemaker patients toward their pacemaker has received some attention in the medical literature. Early studies by Green and Moss, ${ }^{1}$ Blacher and Basch, ${ }^{2}$ and periodic reports by others $\mathrm{s}^{3,4}$ have revealed little in the way of severe anxiety of patients as a result of their dependence on a mechanical device to assist cardiac function. However, physicians caring for patients with permanent pacemakers not infrequently detect anxiety about cardiac function in general, and Goldman et al., have specifically commented on the anxiety which may accompany pulse generator replacement and the implicit dependence that having a pacemaker entails. ${ }^{5}$ It is the purpose of this report to present our experience in the exploration of psychosocial factors in patients with a chronically implanted permanent pacemaker,

Address for reprints: Arthur B. Simon, M.D., 3200 Burnet Avenue, Cincinnati, Ohio 45229 and to describe a dramatic case where chronic depression in a pacemaker patient led to an unsuccessful suicide attempt by physical abuse to the pacing system.

\section{Case History}

On August 30, 1978 a 53-year-old woman was seen in the Emergency Room at University Hospital for a suicide attempt.

She had a long and complicated pacemaker history, having received her initial implant elsewhere in September 1968 for complete AV block following a presumed myocardial infarction. She had received pulse generator replacements in March 1971, May 1971, June 1972, January 1973, January 1975, October 1975, and May 1977. Numerous additional hospitalizations had been required for pulmonary embolus, infected implant sites, and removal of previously infected electrodes. 
She had been under intermittent psychiatric care since 1964 for depression and chronic anxiety. In April 1974, while on a medical program including chlordiazepoxide (Librium-Roche) $100 \mathrm{mg} /$ day for anxiety and methyprylon (Noludar-Roche) 1.20 grams each night for insomnia, she was hospitalized for methyprylon overdose.

She remained relatively stable until one year prior to admission when she reluctantly moved with her family to a more rural setting. She experienced a rapid 40-pound weight loss due to continuing depression and anorexia. Her initial evaluation at the University of Michigan on $11 / 4 / 77$ revealed a cachectic middle-aged woman, 64 inches tall, weighing only 86 pounds. Her cardiac evaluation indicated moderate mitral insufficiency and cardiomegaly. Her pacemaker was functioning properly. She was found to be pacemaker dependent, as defined by a sixsecond pause with pacemaker inhibition.

Her depression continued. Suicidal gestures occurred in January and May 1978. On August 30,1978 , she became despondent, ingested an unknown number of methyprylon capsules, and proceeded to use a razor blade to inflict mul-

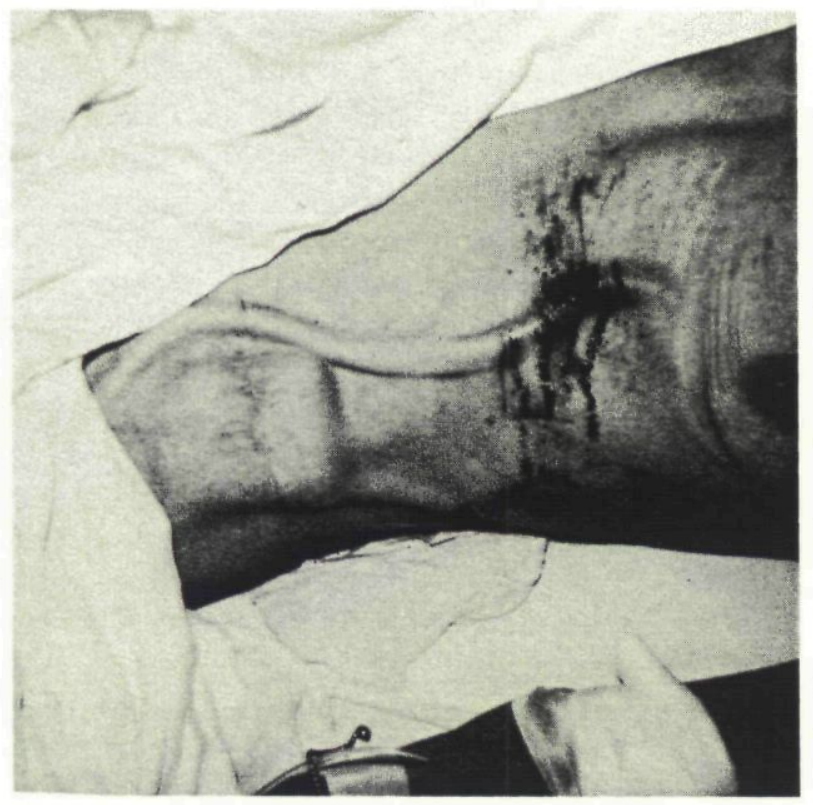

Figure 1. The patient as she presented in the emergency rom. Note the multiple self-inflicted lacerations across the pacing leads. tiple superficial and deep lacerations across her abdominal wall over the subcutaneously implanted epicardial leads (Fig. 1). In the emergency room the electrocardiogram showed normal pacemaker function. Exploration of the wounds demonstrated that the lacerations were down to the pacemaker leads, and at one site incised the endothialized sheath around one lead and had nicked its silastic insulation.

The multiple incision sites were cleansed, dressed with antibiotic ointment and the patient was begun on cephazolin (Kefzol-Lilly) 1 gram intravenously q $6 \mathrm{~h}$ and parenteral fluids. Over a two-week period of hospitalization, initially in the intensive care unit and subsequently in a psychiatric unit, the multiple wounds healed, although a minor degree of cellulitis around the deepest incision persisted for over three weeks. At no time did she become febrile, nor were any arrhythmias noted. Cephalothin (Keflin-Lilly) $500 \mathrm{mg}$, q.i.d. and daily dressing changes were continued.

Psychiatric evaluation revealed that this woman's suicide gesture was precipitated by an acute financial loss and an exacerbation of chronic severe marital discord. There was a long history of drug abuse and dependence antedating her original pacemaker placement, suggesting the strong possibility of a borderline personality disorder and perhaps a propensity to severe endogenous depression at times of severe narcissistic losses. In the psychiatric report these other situational concerns were of paramount importance, and there was no data to suggest that the pacemaker had taken on a delusional importance for her. During her stay in the psychiatric hospital she did not talk of her concerns about pacemaker function.

Two months later the incisions had healed entirely and normal pacemaker function was still present (Fig. 2).

\section{Data}

This dramatic case prompted us to review our own findings in regard to the psychosocial aspects of patients with cardiac pacemakers. One of us (PK) interviewed 30 randomly selected patients in the outpatient pacemaker 


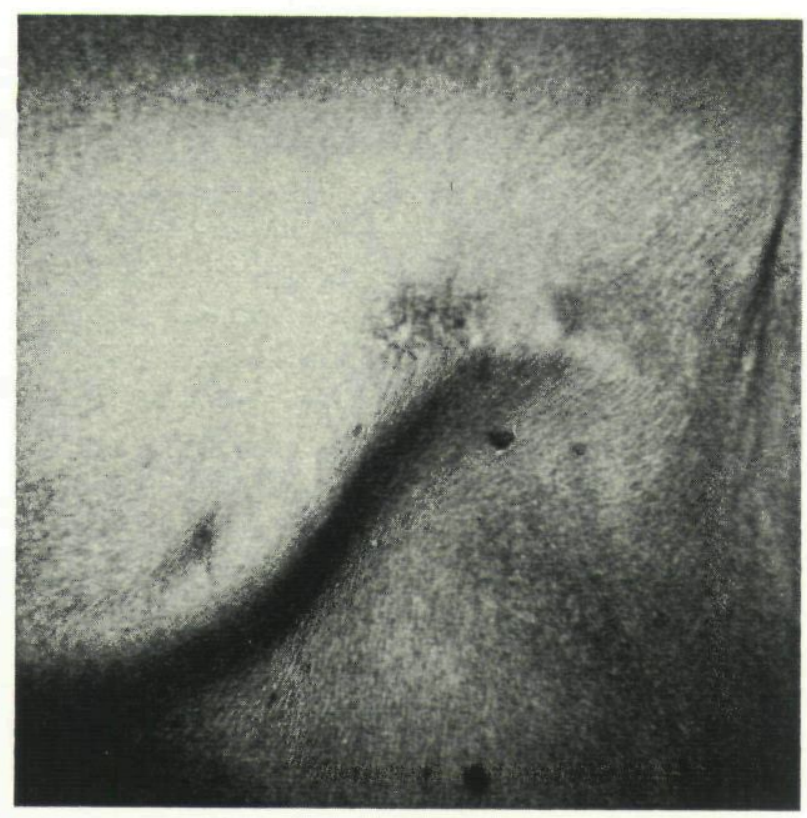

Figure 2. The healed wounds two months later.

clinic to gain an understanding of how psychological symptoms in these patients related to their cardiac condition and their pacemaker. Several patterns of responses were observed.

Some patients joked in the face of ongoing fears. One middle-aged patient with a prosthetic valve and pacemaker referred to himself as "the million dollar man"; a young man in his twenties called himself "Jack the computer." Others entertained fantasies of unrealistic degrees of benefit obtained from the device. A seventy-three-year-old woman asked, "I won't ever be able to die now. Is that true?" Another man half-seriously imputed extra powers to his pacemaker, with the fantasy that it might protect him from developing cancer.

Eighteen patients $(60 \%)$ stated unequivocally that they did not think about the pacemaker or its implications and were generally unconcerned about its presence. In four patients (13\%) the pacemaker appeared to meet certain dependency needs by providing total external responsibility for what happens. Four patients expressed a quiet resignation in terms such as "thank the Lord." In six patients $(20 \%)$, how- ever, there was a veiled depression or even a wish to die; further exploration of these symptoms revealed them to be more related to matters of social isolation, recent losses, and other common issues of old age or enduring character problems than to the pacemaker specifically. In no patient was there any significant overt anxiety about the pacemaker itself or any adverse thoughts about a foreign body in the chest. No patient interviewed expressed overt suicidal tendencies and no patient expressed anxiety about the risk of being totally pacemaker dependent (i.e., that malfunction of the pacing system might or could result in death) despite the fact that many had experienced return of severe symptoms with previous pacemaker system failure.

\section{Discussion}

There has been some concern in recent years with the psychological effects of a variety of artificial devices. Much experience has been gained with dialysis machines, ${ }^{6}$ fetal monitoring, ${ }^{7}$ penile prosthesis, ${ }^{8}$ and other devices, as well as with patients with permanent cardiac pacemakers. In this latter group, despite the relief from frightening symptoms of Stokes-Adams attacks, concern has been expressed that the dependence on an implanted device and an easily palpable and visible foreign body in the chest wall might have acute or long term psychological consequences. These patients face the constant possibility of leads malfunctioning, invariable battery depletion, and government ordered recalls, as well as the constant reminder that their own bodies are no longer functioning properly. Although many patients expressed feelings similar to those seen in others with heart disease, in general these patients were well defined against anxiety, grief or hostility toward their implanted device despite their knowledge of the serious nature of their heart disease. In fact, it is our experience that some patients are especially interested in the pacemaker's operating characteristics, especially those patients with mechanical or engineering backgrounds.

Review of our own data on the natural history of over 490 patients who received a pacemaker 
implant at this institution between 1961-1979 $9^{9,10}$ reveals only one other patient who committed suicide following pacing system replacement.

Pacemaker twiddling, ${ }^{11}$ a form of pacemaker system manipulation which may result in pacing system malfunction, is an uncommon and subtle form of pacemaker system abuse. It is more likely related to idiosyncratic behavior combined with local discomfort or technical factors than due to a specific psychological conflict with the device itself.

The patient reported here had a psychiatric history of repeated severe depression and anxiety antedating her initial pacemaker implantation. The recent weight loss, depression, hopelessness and anorexia were consistent with accepted criteria for a severe "endogenomorphic" depression..$^{12}$ There was no evidence that the pacemaker was the nidus of concern for this patient, although one must assume she attacked her implantation site with the idea of ending her life at its mechanically most vulnerable point. This lady's delusional concern about the hopelessness of her life found its dramatic expression at her pacemaker site.

Her presentation re-emphasizes the importance of recognizing and treating underlying psychiatric syndromes and character problems regardless of their setting. The tell-tale symptoms of severe loss of self-esteem, hopelessness about the future, weight loss, anorexia and insomnia, agitation and mood change may be detected with even a brief patient contact. Referral to a psychiatrist comfortable in working with medical problems may be critical. The physician in a pacemaker clinic is in a good position to screen for the harbingers of severe depression. Many patients do not go of their own accord to a psychiatrist for help with these symptoms.

Our findings also suggest that concerns about psychosocial adjustment of the majority of patients with permanent pacemakers are general-

\section{References}

1. Green, W.A. and Moss, A.J.: Psychosocial factors in the adjustment of patients with permanently implanted cardiac pacemakers. Ann. Intern. Med., 70:897, 1969. ly not related to the pacemaker itself. However, as was also noted in another series, ${ }^{2}$ a significant number of patients in a pacemaker clinic may be classified as clinically depressed, a condition known to be more common in the geriatric population. Patients evaluated in this study typically used denial as seen in their jokes, fantasies and questions in a healthy, adaptive way to keep from unacceptable helpless fears of the future. Joking was commonplace with the theme being the new-found omnipotence of an artificial device which protects against total impotence without it. This was a psychologically determined, but adaptive manner of dealing with pacemaker anxieties. The continuity provided by an empathic physician and specialized nursing personnel was paramount, providing the patient with a reassuring feeling that the pacemaker was a direct line to needed support. A comprehensive patient education program, initiated by the nursing staff as soon as a patient becomes identified as a pacemaker recipient and continued it in the outpatient setting has been an integral part of the pacemaker program at our institution for many years. We feel this instructional program has been responsible for the absence of the "pacemaker panic" described by Goldman et al. ${ }^{5}$ Other similar programs ${ }^{13}$ have been described with equally good results.

In the population studied it became apparent that psychological issues in the pacemaker population were present but universally were understandable in terms of current and chronic psychosocial factors and a life-long character style, emphasizing the importance of proper attention being paid to these. Basically, there was support for Green's assertion ${ }^{1}$ that in this generally geriatric population, the pacemaker is helpful psychologically as well as medically. It provides a concrete and measurable reassurance that life can be extended without the overwhelming fear of recurrent symptomatic arrhythmias.
2. Blacher, R.S. and Basch, S.H.: Psychological aspects of pacemaker implantation. Arch. Gen. Psychiat., 22:319, 1970.

3. Hesse, K.A.F.: Meeting the psychosocial needs of 
pacemaker patients. Int. J. Psychiat. 6:359, 1975.

4. Rodstein, J., Zanct, S., Savitsky, E., and Goldfeder, M.: Relation of long-term electronic cardiac pacing to mental status and adaptation in the institutional aged. J. Amer. Geriatr. Soc., 25:534, 1977.

5. Goldman, B.S., Noble, E.J. and MacGregor, D.C.: Pacemaker panic. Amer. J. Cardiol., 30:705, 1972.

6. Steele, T.E., Finklestein, S.H., and Finklestein, F.: Hemodialysis patients and spouses. J. Nerv. Ment. Dis., 162:225, 1976.

7. Starkman, M.: Psychological responses to the use of the fetal monitor during labor. Psychosom. Med., 38:269, 1976.

8. Stewart, T.D.: Penile prosthesis: Psychologic fac- tors. Urology, 7:400, 1976.

9. Simon, A.B. and Zloto, A.E.: Atrioventricular block: Natural history after ventricular pacing. Amer. J. Cardiol., 41:500, 1978.

10. Simon, A.B. and Zloto, A.E.: Symptomatic sinus node disease: Natural history after permanent ventricular pacing. PACE, 2:305, 1979.

11. Bayliss, C.E., Beanlands, D.S., and Baird, R.J.: The pacemaker-twiddler's syndrome: A new complication of implantable transvenous pacemakers. Can. Med. J., 99:371, 1968.

12. Klein, D.F.: Endogenomorphic depression. Arch. Gen. Psychiat., 31:447, 1974.

13. Hesse, K.A.F.: Meeting the needs of the pacemaker patients. Int. J. Psychiat. Med., 6:359, 1975.

\section{FIRST MEETING OF THE NORTH AMERICAN SOCIETY OF PACING AND ELECTROPHYSIOLOGY}

Whitehall Hotel, Houston, Texas

Thursday, March 13, 1980, 1:00 to 5:00 P.M.

Program:

I. Panel Discussion - Cardiac Pacing and Tachyarrhythmias (1:00-3:00)

Chairman: Douglas P. Zipes, M.D.

Participants: John D. Fisher, M.D., John Gallagher, M.D., Mark Josephson, M.D., Kenneth Rosen, M.D.

II. Keynote Address - Perspectives in Cardiac Pacing-1980

(3:00-3:30)

Victor Parsonnet, M.D.

III. Break

(3:30-4:00)

IV. Business Meeting

(4:00-5:00) 
This document is a scanned copy of a printed document. No warranty is given about the accuracy of the copy. Users should refer to the original published version of the material. 